\title{
MicroRNA-29a inhibits mesenchymal stem cell viability and proliferation by targeting Roundabout 1
}

\author{
YUDONG ZHANG and SHENGHUA ZHOU \\ Department of Cardiology, The Second Xiangya Hospital, Central South University, Changsha, Hunan 410011, P.R. China
}

Received March 11, 2014; Accepted November 20, 2014

DOI: $10.3892 / \mathrm{mmr} .2015 .4183$

\begin{abstract}
Secreted Slit glycoproteins and their Roundabout (Robo) receptors have been identified as important axon guidance molecules. The pivotal role of Slit-Robo signaling is in regulating cell proliferation. MicroRNAs (miRNAs), a class of small non-coding RNAs, function as critical regulators of gene expression by binding to the 3'-untranslated region of mRNAs and causing mRNA degradation or translational repression. The present study demonstrated that downregulation of Robo1 using small interfering RNA inhibited mesenchymal stem cell (MSC) proliferation. Additionally, four miRNAs (miR), including miR-218, miR-29a, miR-146 and miR-148, inhibited the protein expression of Robol in the MSCs, with miR-29 having the most marked effect. A luciferase reporter assay identified Robol as a novel target of miR-29a. Overexpression of miR-29a suppressed the protein expression levels of Robol and Slit2 and inhibited the viability and proliferation of the MSCs. By contrast, overexpression of Robo1 partly rescued these inhibitory effects of miR-29a on the MSCs confirming that miR-29a inhibited MSC viability and proliferation, at least partially, by directly targeting Robol. These results indicated that the miR-29a/Robo1 axis is crucial for the regulation of MSC viability and proliferation, suggesting that miR-29a may serve as a potential clinical target for MSC expansion and stem cell transplantation.
\end{abstract}

\section{Introduction}

Stem cell transplantation has been investigated extensively as a therapeutic approach to regenerate tissues following injury (1). Mesenchymal stem cells (MSCs), which can be readily isolated and expanded, have neurogenic, chondrogenic, adipogenic, osteogenic and myogenic properties under specific

Correspondence to: Professor Shenghua Zhou, Department of Cardiology, The Second Xiangya Hospital of Central South University, 139 Renmin Middle Road, Changsha, Hunan 410011, P.R. China

E-mail: csuzhoushenghua@163.com

Key words: mesenchymal stem cell, proliferation, Roundabout 1, microRNA-29a differentiating conditions (2). Furthermore, MSCs are genetically stable with have a low risk of immune rejection and, therefore, are often used as seeding cells in tissue engineering and in stem cell therapy (3).

The ability to accurately regulate of cell fate determination is a prerequisite for the future therapeutic use of MSCs. MicroRNAs (miRNAs), a class of small non-coding RNAs, negatively regulate the expression of a variety of genes (4). miRNAs affect diverse cellular pathways by inducing RNA interference-based mechanisms at a post-transcriptional level (5). Based on the complicated association between miRNAs and the mRNA 3'-untranslated region (UTR), several online tools have been developed, including TargetScan, PicTar and TargetRank, which provide miRNA target predictions based on sequence complementarity in the optimal base-pairing of miRNA to the seed region and sequence conservation (6-8).

Roundabout 1 (Robo1) is expressed in multiple cell types, including embryonic stem cells, cadiocytes, vascular endothelial cells and MSCs $(9,10)$. In addition, Robol is crucial in the regulation of cell proliferation, migration and differentiation $(11,12)$. Inhibition of the expression of Robol has a suppressive effect on cell proliferation $(11,13)$, however, the role of Robol in MSCs remains to be fully elucidated.

In order to identify novel miRNAs, which target Robol, the present study used bioinformatic predications and confirmed the association between miR-29a and Robo1 by performing luciferase reporter assays.

\section{Materials and methods}

Cell transfection. Human MSCs were purchased from the American Type Culture Collection (Manassas, VA, USA) and were cultured in RPMI-1640 medium (Invitrogen Life Technologies, Carlsbad, CA, USA) supplemented with $10 \%$ fetal bovine serum (Gibco-BRL, Carlsbad, CA, USA) at $37^{\circ} \mathrm{C}$ in a humidified atmosphere with $5 \% \mathrm{CO}_{2}$. To achieve overexpression of miR-29a, the cells were transfected with an miR-29a lentivirus (Genepharma Co., Ltd., Shanghai, China) using Lipofectamine 2000 (Invitrogen Life Technologies). The overexpression of Robol was achieved using a Robol open reading frame-expressing clone (GeneCopoecia, Guangzhou, China). The cells were plated in 6-well plates or 96-well plates, transfected and incubated at $37^{\circ} \mathrm{C}$ for 24 or $48 \mathrm{~h}$ prior to their use in subsequent assays or for RNA and protein extraction. 
Construction of lentiviral vectors overexpressing miR-29a and Robol. To produce a lentivirus expressing mature miR-29a, the pre-miRNA sequence was synthesized and a control scrambled construct (control RNAi) with no homology to the human genome was designed (5'-AAT GTA CTG CGC GTG GAG A-3'). The sequences were cloned into the $\mathrm{HpaI}$ and XhoI sites of pGCSIL-green fluorescent protein (GFP) (GeneChem, Shanghai, China) to produce the pGCSIL-GFP-miR-29a or pGCSIL-GFP-control, respectively. Small interfering (si)RNA targeting Robol was purchased from Auragene Bioscience, Inc. (Changsha, China).

Lentivirus production, titration and infection. To produce the miR-29a and the control lentivirus, the plasmids encoding either miR-29a or the control scrambled sequence were cotransfected into 293T cells with the pHelper1.0 and pHelper 2.0 plasmids (GeneChem), which contained elements required for virus packaging, using Lipofectamine 2000 (Invitrogen Life Technologies) according to the manufacturer's instructions. The culture supernatants containing the lentivirus were harvested, ultra-centrifuged at 70,000 x g for $90 \mathrm{~min}$, and the viral titers were determined using plaque-based assays. For lentiviral infection, the target cells were plated at $40-50 \%$ confluency and incubated overnight for $16 \mathrm{~h}$ at $37^{\circ} \mathrm{C}$. The culture medium, consisting of Dulbecco's modified Eagle's medium (Cellgro, Manassas, VA, USA) containing 10\% fetal bovine serum (Gibco-BRL), was then replaced with viral supernatant $(1.5 \mathrm{ml} /$ well $)$ and incubated at $37^{\circ} \mathrm{C}$ for $10 \mathrm{~h}$, prior to replacement of the viral supernatant with fresh media. After $48 \mathrm{~h}$ incubation, the infected cells were selected using puromycin ( $2 \mathrm{mg} / \mathrm{ml})$ (InvivoGen, Toulouse, France) for 5 days.

$R N A$ extraction and reverse transcription quantitative polymerase chain reaction ( $R T-q P C R)$. The MSCs were seeded $\left(\sim 1.0 \times 10^{6}\right)$ into 6 -well culture plates for $72 \mathrm{~h}$ prior to harvesting. Small RNAs ( 200 nt) were isolated using a mirVana ${ }^{\mathrm{TM}}$ PARIS $^{\mathrm{TM}}$ kit (Ambion ${ }^{\circledR}$ Life Technologies, Carlsbad, CA, USA) according to the manufacturer's instructions. For the RT reactions, $1 \mathrm{mg}$ small RNAs were reverse transcribed using an miScript RT kit (Qiagen, Hilden, Germany) at $37^{\circ} \mathrm{C}$ for $60 \mathrm{~min}$ followed by a final incubation at $95^{\circ} \mathrm{C}$ for $5 \mathrm{~min}$. RT-qPCR was performed using an miScript SYBR Green PCR kit (Qiagen) on a CFX96 real-time PCR machine (Bio-rad Laboratories, Inc., Hercules, CA, USA). The qPCR was performed at $95^{\circ} \mathrm{C}$ for $15 \mathrm{~min}$, followed by 40 cycles at $94^{\circ} \mathrm{C}$ for $15 \mathrm{sec}, 55^{\circ} \mathrm{C}$ for 30 sec and $70^{\circ} \mathrm{C}$ for $30 \mathrm{sec}$. The expression level of each miRNA was normalized to U6 short non-coding RNA.

The mRNA expression level of Robol was detected by RT-qPCR using a SYBR Green RT-PCR kit (Bio-Rad Laboratories, Inc.) according to the manufacturer's instructions. Briefly, the total RNA was extracted from the cells using TRIzol reagent (Invitrogen Life Technologies) and the cDNA was synthesized using a RevertAid First-Strand cDNA Synthesis kit (Fermentas, Pittsburgh, PA, USA), according to the manufacturer's instructions. Each cDNA sample was used as a template for the qPCR in triplicate using iQTM SYBR Green Supermix (Bio-Rad Laboratories, Inc.) by denaturation at $94^{\circ} \mathrm{C}$ for $1 \mathrm{~min}, 30$ cycles at $94^{\circ} \mathrm{C}$ for $40 \mathrm{sec}$ and $60^{\circ} \mathrm{C}$ for $40 \mathrm{sec}$, followed by extension at $72^{\circ} \mathrm{C}$ for $6 \mathrm{~min}$. The specific primer pairs were: Robol (107 bp), sense 5'-GGC GGT GAA
GGA GAT GAA C-3' and antisense 5'-TGA TGA GGA AAT CCA CGA TAG AG-3' and $\beta$-actin (202 bp), sense 5'-GGC G GC ACC ACC ATG TAC CCT-3' and antisense 5'-AGG GG C CGG ACT CGT CAT ACT-3'. The relative mRNA expression levels of Robol were normalized to the internal control, $\beta$-actin. The relative gene expression was quantified using CFX Manager version 1.6 software (Bio-Rad Laboratories, Inc.) and data were expressed as a percentage compared with the control cells.

Western blotting. The cells were cultured in $35 \mathrm{~mm}$ dishes prior to lysis in $0.2 \mathrm{ml}$ lysis buffer containing $0.1 \%$ SDS, $1 \%$ NP-40, 50 mM HEPES (pH 7.4), 2 mM EDTA, $100 \mathrm{mM} \mathrm{NaCl}$, $5 \mathrm{mM}$ sodium orthovanadate, $40 \mu \mathrm{M}$ p-nitrophenyl phosphate and $1 \%$ protease inhibitor mixture set I (Calbiochem, La Jolla, CA, USA). The lysates were centrifuged at 12,000 rpm for $15 \mathrm{~min}$ and the supernatants were collected, denatured and separated using 10\% SDS-PAGE gels and blotted onto polyvinylidene difluoride membranes. The membranes were then blocked in $5 \%$ bovine serum albumin for $1.5 \mathrm{~h}$ at room temperature, followed by incubation overnight at $4^{\circ} \mathrm{C}$ with the following antibodies: Mouse anti-human monoclonal anti-BrdU (B2531; dilution 1:1,000; Sigma-Aldrich, St. Louis, MO, USA) and polyclonal mouse anti- $\beta$-actin (sc-47778; dilution 1:2,000; Santa Cruz Biotechnology, Inc., Dallas, TX, USA). The membranes were rinsed and incubated for $1 \mathrm{~h}$ with goat anti-mouse HRP-conjugated secondary antibodies (sc-2005; dilution 1:5,000; Santa Cruz Biotechnology, Inc.). Chemiluminescent detection was performed using an Enhanced Chemiluminescence Detection kit (Pierce Biotechnology, Inc., Rockford, IL, USA).

Luciferase reporter assay. A luciferase reporter assay was performed in human embryonic kidney 293a (HEK293a) cells (Huiying Biological Technology Co., Ltd., Xian, China). Vectors, based on either pMIR-REPORT, containing the wild-type (WT) $350 \mathrm{bp}$ fragment of the Robo1 3'-UTR or the same fragment with mutation (MUT) of the miR-29a binding site (199-194 bp), were inserted downstream of the luciferase reporter gene stop codon in pMIR-REPORT using HindIII and SpeI sites. The cells were cotransfected with either the miR-29a lentivirus, the miR-67-negative-control lentivirus (50 $\mathrm{nM})$, the pMIR-REPORT vectors containing the WT or MUT miR-29a binding sites (400 ng) or pRL-SV40 (Promega Corporation, Madison, WI, USA) expressing Renilla luciferase (400 ng) for normalization of the transfection efficiency. The cells were grown in high-glucose Dulbecco's modified Eagle's medium supplemented with $10 \%$ fetal bovine serum and the luciferase activities were measured $48 \mathrm{~h}$ after transfection using a Dual-Luciferase Reporter assay system (Promega Corporation).

MTT assay. The cell viability was evaluated using a modified MTT assay. The MSCs were seeded $\left(2 \times 10^{3}\right)$ into 96-well plates and the viability of the cells transfected with either the miR-29a or control were assessed at five time points (days 1-5). Briefly, the quantification of mitochondrial dehydrogenase activity was determined via the enzymatic conversion of MTT (Sigma-Aldrich) to a colored formazan product. MTT $(10 \mu \mathrm{l} ; 10 \mathrm{mg} / \mathrm{ml})$ was added to the cells and incubated 
A

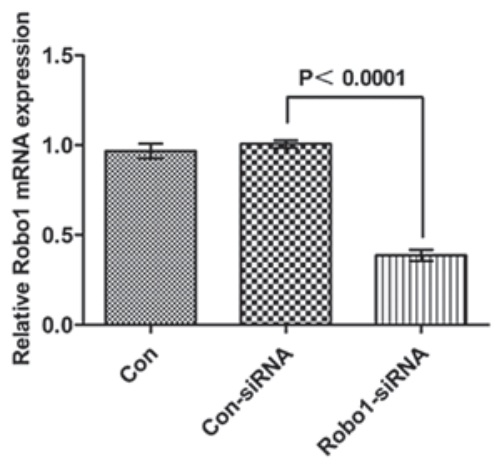

B
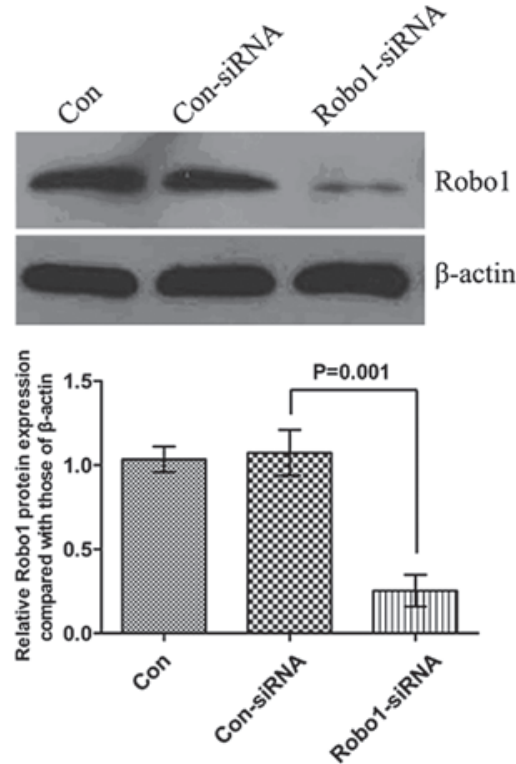

C

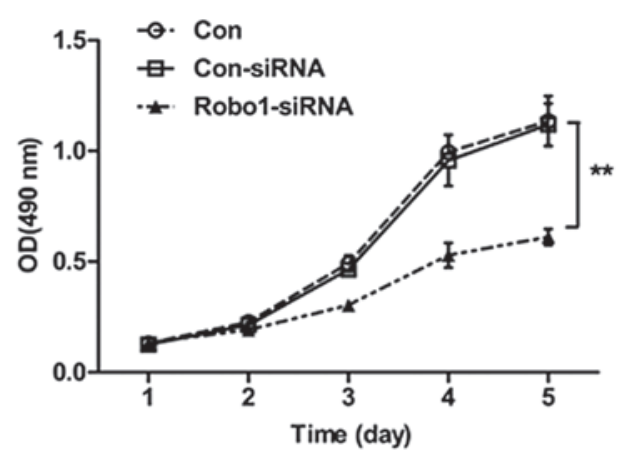

D

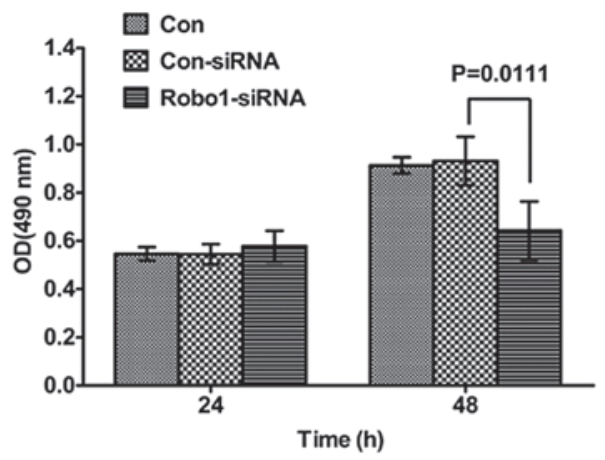

Figure 1. Downregulation of Robol using siRNA inhibits the cell viability and proliferation in MSCs. Following transfection of Robol-siRNA or Con-siRNA into MSCs, the (A) mRNA expression level of Robol was determined by performing reverse transcription quantitative polymerase chain reaction; (B) protein levels of Robol were determined by western blotting; (C) cell viability was determined by performing an MTT assay ( $(* \mathrm{P}<0.01)$ and (D) cell proliferation was assessed using a 5-bromo-2-deoxyuridine incorporation assay. Data are expressed as the mean \pm standard deviation. siRNA, small interfering RNA; Con, control nontransfected cells; Con-siRNA, cells transfected with scramble siRNA; Robol, roundabout 1; MSC, mesenchymal stem cell; OD, optical density.

for $4 \mathrm{~h}$ prior to termination of the reaction by removing the supernatant and additing $100 \mu 1$ dimethylsulfoxide to dissolve the formazan product. Following incubation for $30 \mathrm{~min}$, the optical density of each well was measured at $570 \mathrm{~nm}$ using a plate reader (ELx808; BioTek Instruments, Inc., Winooski, VT, USA).

5-Bromo-2-deoxyuridine (BrdU) incorporation assay. The DNA synthesis in the proliferating cells was determined by measuring BrdU incorporation. BrdU assays were performed at 24 and $48 \mathrm{~h}$ after the transfection of the MSCs with the miR-29a or control vectors. The transfected cells were seeded $\left(2 \times 10^{3}\right)$ into 96 -well plates, cultured for 24 or $48 \mathrm{~h}$ and incubated with a final concentration of $10 \mu \mathrm{M}$ BrdU (BD Pharmingen, San Diego, CA, USA) for $2-24 \mathrm{~h}$ at $37^{\circ} \mathrm{C}$. Following incubation, the medium was removed and the cells were fixed with 4\% paraformaldehyde (Amsbio, Beijing, China) for $30 \mathrm{~min}$ at room temperature, prior to incubation with a peroxidase-coupled monoclonal mouse anti-human BrdU-antibody (1:1,000; Sigma-Aldrich) for $60 \mathrm{~min}$ at room temperature. The cells were then washed three times with phosphate-buffered saline, incubated with tetramethylbenzidine for $30 \mathrm{~min}$ and the absorbance values were measured at $490 \mathrm{~nm}$ using the ELx808 microplate reader. The background BrdU immunofluorescence was determined using BrdU-negative cells stained with the BrdU antibody.

Statistical analysis. Data are expressed as the mean \pm standard deviation of three independent experiments and processed using SPSS 17.0 software (SPSS, Inc., Chicago, IL, USA). The expression of miR-29a in the MSC tissues and the paired adjacent normal colonic tissues were compared using Wilcoxon's paired test. The differences between the groups in the migration and invasion assays were evaluated by one-way analysis of variance. $\mathrm{P}<0.05$ was considered to indicate a statistically significant difference.

\section{Results}

Downregulation of Robol using siRNA inhibits the viability and proliferation of MSCs. To determine the potential role for Robol in MSCs, Robo1-specific siRNA was transfected into MSCs to suppress the expression of Robol. Following transfection, the mRNA and protein levels of Robol in the MSCs were determined. The mRNA and protein levels were notably downregulated following transfection with Robo1-siRNA 
A

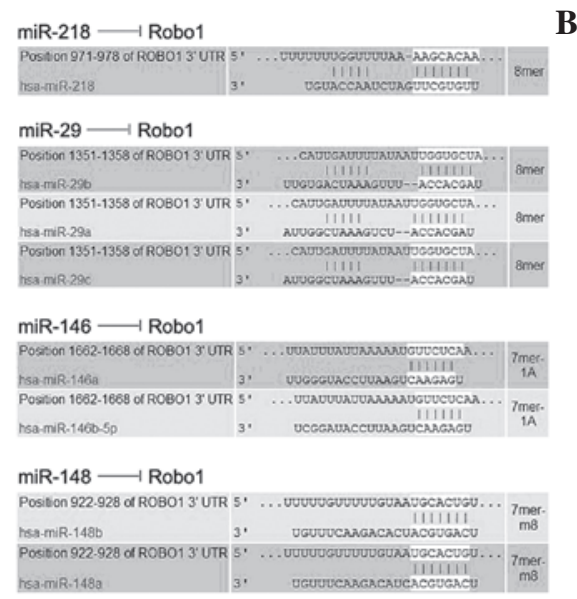

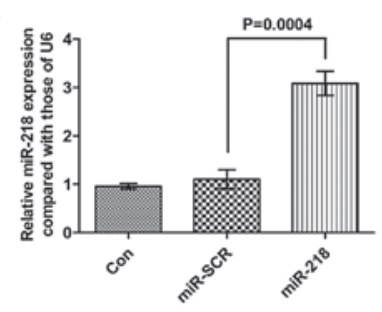
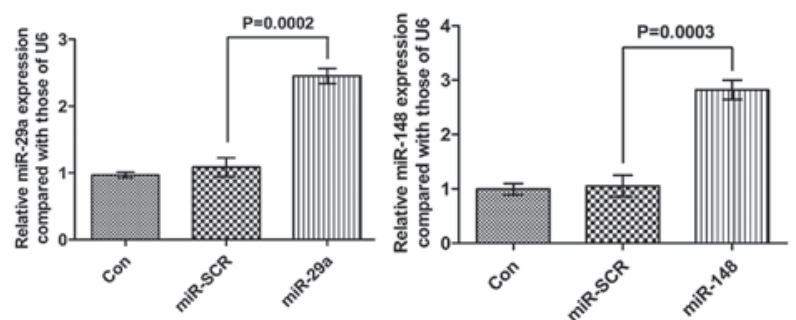

\section{C}

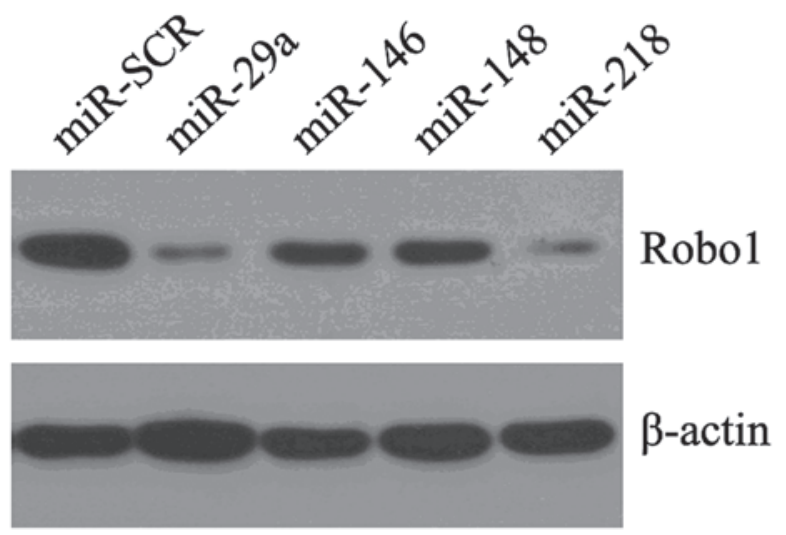

Figure 2. miR-29a inhibits the protein expression of Robol in MSCs. (A) Targetscan was used to predict which miRNAs directly targeted Robo1. Following transfection of MSCs with miR-218, 29a, 146 and 148 mimics, the (B) mRNA expression levels were determined by reverse transcription quantitative polymerase chain reaction. Data are expressed as the mean \pm standard deviation. (C) Protein levels of Robol were examined using western blotting. miR, microRNA; Con, control untransfected cells; Robo1, Roundabout 1; MSC, mesenchymal stem cell; SCR, scramble lentivirus.

(Fig. 1A and B), indicating that the transfection efficiency was satisfactory. The MTT and BrdU incorporation assays were subsequently used to determine the effects of Robol downregulation on MSC viability and proliferations. As shown in Fig. $1 \mathrm{C}$ and D, the viability and proliferation of the Robo1-siRNA-transfected MSCs were reduced compared with the non-transfected cells or those transfected with the control siRNA, indicating that the inhibition of Robol suppressed the viability and proliferation of the MSCs.

miR-29a markedly inhibits the protein expression of Robol in MSCs. To examine the regulatory effects of miRNAs on the expression of Robol in the MSCs, bioinformatical predication was performed. Several putative miRNAs were identified, including miR-218, miR-29a, miR-146 and miR-148 (Fig. 2A). The MSCs were then transfected with the identified miRNA mimics. Following transfection, the expression levels of the four miRNAs were determined using RT-qPCR, all of which were significantly increased (Fig. 2B), indicating a satisfactory transfection efficiency. Subsequently, western blotting was performed to examine the protein expression of Robo1. All four miRNAs inhibited the protein expression of Robol, however, miR-29a had the most marked effect (Fig. 2C).

Robol mRNA is a direct target of miR-29a. To confirm the hypothesis that miR-29a binds to the 3'-UTR of Robol mRNA
(Fig. 3A), a WT Robo1 3'-UTR luciferase reporter vector (WT-Robo1) and a mutated version of the Robo1 3'-UTR luciferase reporter vector (MUT-Robol) were produced by sequentially mutating the predicted $8 \mathrm{bp}$ miR-29a binding site of the Robo1 3'-UTR (Fig. 3A). The WT-Robol vector was cotransfected with either the miR-29a lentivirus or the scrambled control into the HEK293 cells. The luciferase activity of the Robo1 3'-UTR luciferase reporter vector was significantly reduced in the miR-29a-transfected cells compared with the scrambled control cells (Fig. 3B). In addition, the miR-29a-mediated repression of Robol 3'-UTR luciferase reporter activity was eliminated by mutation of the putative miR-29a binding site in the Robol 3'-UTR (Fig. 3B).

Overexpression of miR-29a inhibits the expression of Slit2 and Robol in MSCs. To investigate the role of miR-29a in MSCs, the MSCs were infected with an miR-29a or miR-scramble lentivirus. RT-qPCR revealed that miR-29a was significantly upregulated in the MSCs infected with the miR-29a lentivirus compared with those infected with the scrambled control lentivirus (Fig. 4A). In addition, overexpression of miR-29a resulted in a significant decrease in the mRNA and protein expression levels of Slit 2 and Robol in the MSCs (Fig. 4B and C). These findings indicated that miR-29a inhibits the regulation of the Slit 2 and Robol expression in the MSCs. 


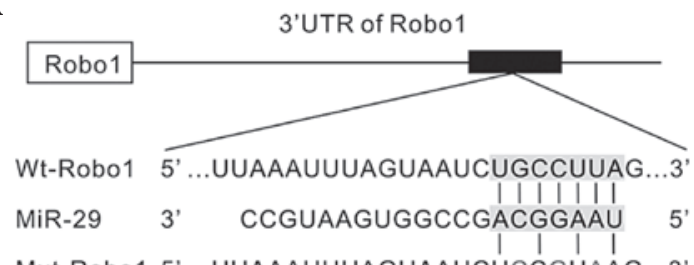

Mut-Robo1 5' ...UUAAAUUUAGUAAUCUCCGUAAG...3'

B

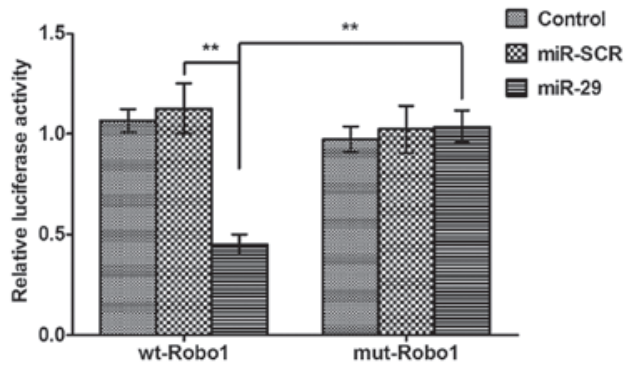

Figure 3. Robol mRNA is a direct target of miR-29a. (A) According to the bioinformatic predictions, Wt-Robo1 and Mut-Robo1 3'-UTR luciferase reporter vectors were generated by sequentially mutating the predicted 8 bp miR-29a binding site in the Robol 3'-UTR. (B) A luciferase reporter assay was performed to determine whether Robo1 mRNA was a direct target of miR-29. The cells were co-transfected with either $50 \mathrm{nM}$ miR-29a or scramble miR-SCR, 400 ng pMIR-REPORT vectors containing the Wt or Mut miR-29a binding sites and 400 ng pRL-SV40 expressing Renilla luciferase for normalization of the transfection efficiency. The luciferase activities were measured using a Dual-Luciferase Reporter assay system $48 \mathrm{~h}$ after transfection $\left({ }^{* *} \mathrm{P}<0.01\right)$. Data are expressed as the mean \pm standard deviation. Robol, Roundabout 1; Wt, wild-type; miR, microRNA; Mut, Mutant; UTR, untranslated region; SCR, scramble lentivirus.

Overexpression of miR-29a inhibits MSC viability and proliferation by targeting Robol. To investigate whether miR-29a regulates MSC viability and proliferation by targeting Robol, the MSCs were infected with either the miR-29a lentivirus, the control or the Robol lentivirus or were co-transfected with the miR-29a and Robol lentiviruses. Prior to performing cell viability and proliferation assays, the efficiency of the overexpression of Robol was determined. As shown in Fig. 5A and B, following infection with Robol lentivirus, the mRNA and protein levels of Robol were markedly upregulated in the MSCs. MTT and BrdU incorporation assays were subsequently performed to determine the cell viability and proliferation of the cells in each group. As shown in Fig. 5C and D, the forced upregulation of miR-29a significantly inhibited MSC viability and proliferation, while overexpression of Robo1 notably promoted the viability and proliferation in the MSCs. In addition, the inhibitory effects of miR-29a upregulation on the viability and proliferation of the MSCs were restored by the overexpression of Robol, indicating that miR-29a inhibited MSC viability and proliferation, at least partially, by directly targeting Robo1 (Fig. 5C and D).

\section{Discussion}

The present study, was the first, to the best of our knowledge, to reveal the crucial role of the miR-29a/Robol axis in the regulation of MSC viability and proliferation. In addition, the results identified that Robol was a direct target of miR-29a and that miR-29a had an inhibitory effect on MSC viability and proliferation, at least partly through direct inhibition of Robol.
A

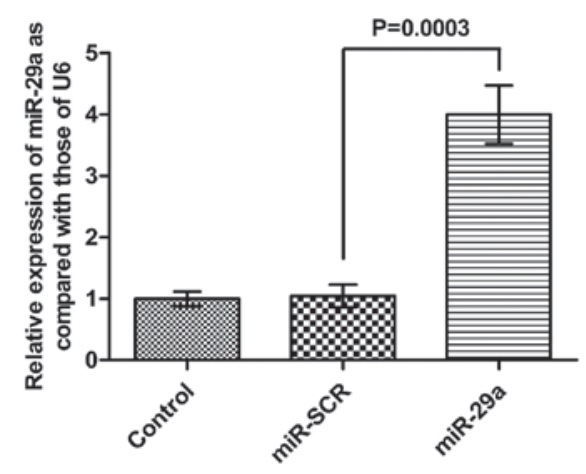

B

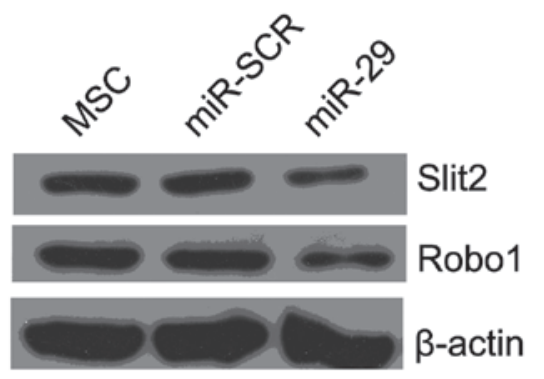

C
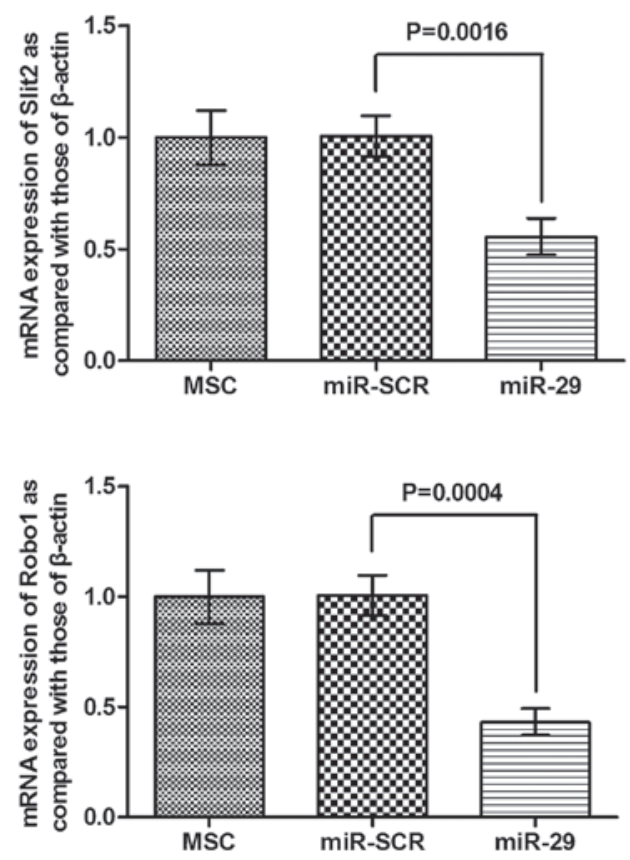

Figure 4. Overexpression of miR-29a inhibits the expression of Slit2 and Robol in MSCs. (A) Following MSC infection with miR-29a or miR-SCR, the mRNA expression of miR-29a was determined by performing RT-qPCR. Data are expressed as the mean \pm standard deviation. (B) Following infection, the protein expression levels of Slit2 and Robo1 were examined by western blotting. (C) Following infection, the mRNA expression levels of Slit2 and Robol were determined using RT-qPCR. Data are expressed as the mean \pm standard deviation. miR, microRNA; MSC, mesenchymal stem cell; Robo1, roundabout 1, RT-qPCR, reverse transcription quantitative polymerase chain reaction; SCR, scramble lentivirus.

Robol has been found to be fundamental in the regulation of multiple biological processes, including cellular proliferation, differentiation, migration, embryonic development, angiogenesis and types of cancer $(10,11,14)$. Several studies have revealed that Slit-Robo signaling is involved in 
A

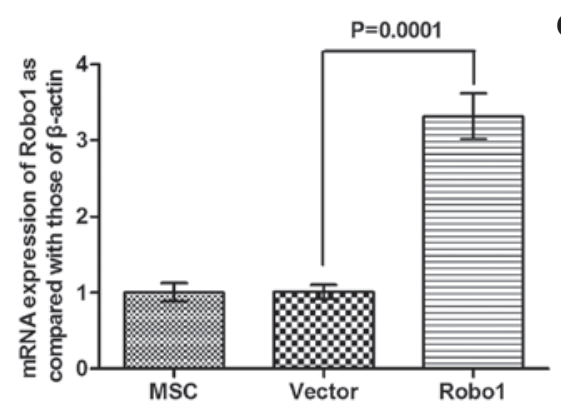

B

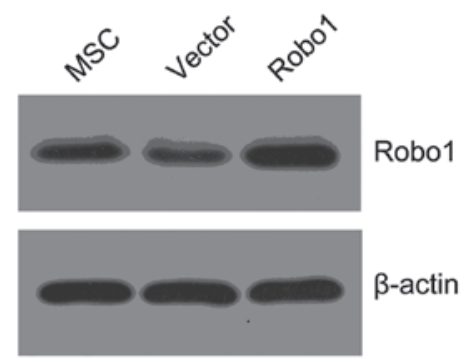

C

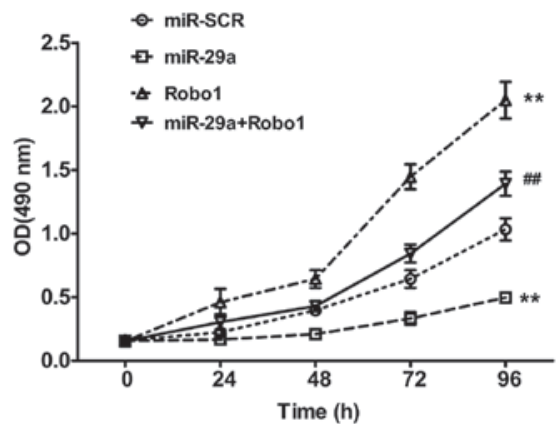

D

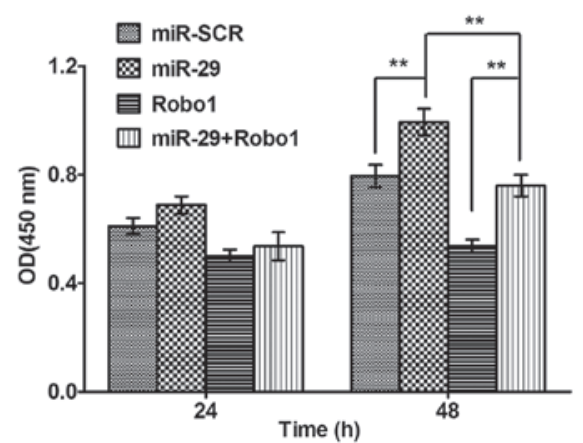

Figure 5. Overexpression of miR-29a inhibits MSC viability and proliferation by targeting Robol. Following MSC infection with the Robol overexpressing lentivirus or blank vector lentivirus, the (A) mRNA level of Robol was determined using reverse transcription quantitative polymerase chain reaction. Data are expressed as the mean \pm standard deviation. (B) protein level of Robol was determined using western blotting. (C) Following MSC infection with miR-29a lentivirus and/or Robol overexpressing lentivirus, an MTT assay was performed to determine the MSC viability in each group. MSCs infected with miR-SCR were used as a control ( ${ }^{\# \#} \mathrm{P}<0.05$, vs. miR-SCR and ${ }^{* *} \mathrm{P}<0.01$, vs. miR-SCR). Data are expressed as the mean \pm standard deviation. (D) Following MSC infection with miR-29a lentivirus and/or Robol overexpressing lentivirus, a BrdU assay was performed to determine the MSC proliferation in each group. MSCs infected with miR-SCR were used as a control ( $\left.{ }^{* *} \mathrm{P}<0.01\right)$. Data are expressed as the mean \pm standard deviation. MSC, mesenchymal stem cell; Robo1, Roundabout 1; miR, microRNA; BrdU, 5-bromo-2-deoxyuridine; OD, optical density.

the regulation of MSC biological processes $(15,16)$. Sun et al demonstrated that the mRNAs of Slit2 and Robol are expressed during the osteoblastic differentiation of MSC-derived cell lines (15). Another previous study revealed that Slit2 cooperated with Robol to maintain Robo-expressing cells, including MSCs in bone marrow niches at steady state and following radiation (16). However, no direct evidence has been found to confirm that Robol is involved in the regulation of MSC viability and proliferation. The present study demonstrated that silencing of Robol by siRNA suppressed the viability and proliferation of MSCs.

miRNAs, as small molecular regulators of gene expression, are important in stem cell function (17). Their ability to fine-tune protein levels is often exploited against key proteins involved in self-renewal or differentiation, including the targeting of Slit/Robo signaling by miR-218 in angiogenesis (18). Additionally, miR-29 has multiple distinct functions and the role of miR-29a in the differentiation of MSC-derived cells has been previously reported. miR-29b initiates the osteogenic signaling by suppressing anti-osteogenic factors, including activin receptor type-2A, catenin, $\beta$-interacting protein 1 , histone deacetylase 4 , transforming growth factor- $\beta 3$ and dual specificity protein phosphatase 2 (19). Guerit et al demonstrated that miRNA-29a is involved in the forkhead box $\mathrm{O} 3$ controlled chondrogenic differentiation of MSCs and cartilage formation (20). miR-29a also acts as a mediator in the $\mathrm{miR}-335-5 \mathrm{p} / \mathrm{sex}$ determining region Y-box 9 controlled chondrogenesis in mouse MSCs (21) and Yan et al reported that miR-29a is involved in the polyhydroxyalkanoate-induced chondrogenic differentiation of MSCs by directly targeting col2a1, which encodes type II collagen (22). Therefore, miR-29a is important in regulating the osteogenic and chondrogenic differentiation of MSCs. However, no other functions on the effects of miR-29a on the biological processes in MSCs have been reported. The present study, was the first, to the best of our knowledge, to demonstrate that miR-29a has an inhibitory effect in the regulation of MSC viability and proliferation.

As Robol was identified as a novel target of miR-29a and had reverse effects on MSC viability and proliferation, the present study hypothesized that miR-29a inhibited MSCs viability and proliferation by directly targeting Robol. To confirm this hypothesis, the present study upregulated the expression levels of miR-29a and/or Robol in MSCs. Upregulation of miR-29a inhibited the protein expression of Robol and inhibited MSC viability and proliferation. The restoration of Robol partially rescued the inhibitory effects of the miR-29a upregulation on the viability and proliferation of the MSCs, which suggested that miR-29a had a suppressive effect in the regulation of MSC viability and proliferation, at least partly via the direct downregulation of Robol. MSCs have also been demonstrated to be regulated by other miRNAs. Chen et al demonstrated that miR-125b suppresses the proliferation and osteogenic differentiation of human bone marrow-derived MSCs (23) and Mao et al demonstrated that miR-23a is involved in tumor necrosis factor- $\alpha$ induced apoptosis in MSCs (24). These findings and those of the present study provide evidence supporting a role for miRNAs in the regulation of MSC biological processes. 
In conclusion, the present study demonstrated that miR-29a inhibited MSC viability and proliferation via the targeting of Robo1. These results suggested that the miR-29a/Robol axis is important in MSCs, raising the possibility that administering miR-29a inhibitors may be used as a therapeutic technique for diseases for the expansion of MSCs prior to transplantation.

\section{References}

1. Bayraktar UD and Ciurea SO: Strategies in haploidentical stem cell transplantation in adults. Turk J Haematol 30: 342-350, 2013.

2. Tanabe S: Role of mesenchymal stem cells in cell life and their signaling. World J Stem Cells 6: 24-32, 2014.

3. Das M, Sundell IB and Koka PS: Adult mesenchymal stem cells and their potency in the cell-based therapy. J Stem Cells 8: 1-16, 2013.

4. Moss EG: MicroRNAs: Hidden in the genome. Curr Biol 12: R138-140, 2002.

5. Sarkar D, Leung EY, Baguley BC, Finlay GJ and Askarian-Amiri ME: Epigenetic regulation in human melanoma: Past and future. Epigenetics: Jan 14, 2015 (Epub ahead of print).

6. Peterson SM, Thompson JA, Ufkin ML, Sathyanarayana P, Liaw L and Congdon CB: Common features of microRNA target prediction tools. Front Genet 5: 23, 2014.

7. Lewis BP, Burge CB and Bartel DP: Conserved seed pairing, often flanked by adenosines, indicates that thousands of human genes are microRNA targets. Cell 120: 15-20, 2005.

8. Krek A, Grun D, Poy MN, et al: Combinatorial microRNA target predictions. Nat Genet 37: 495-500, 2005.

9. Andrews W, Barber M, Hernadez-Miranda LR, et al: The role of Slit-Robo signaling in the generation, migration and morphological differentiation of cortical interneurons. Dev Biol 313: 648-658, 2008.

10. Yuen DA and Robinson LA: Slit2-Robo signaling: A novel regulator of vascular injury. Curr Opin Nephrol Hypertens 22: 445-451, 2013

11. Wang G, Li Y, Wang XY, et al: Slit/Robol signaling regulates neural tube development by balancing neuroepithelial cell proliferation and differentiation. Exp Cell Res 319: 1083-1093, 2013.
12. Dontula R, Dinasarapu A, Chetty C, et al: MicroRNA 203 modulates glioma cell migration via Robo1/ERK/MMP-9 signaling. Genes Cancer 4: 285-296, 2013.

13. Huang L, Xu Y, Yu W, et al: Effect of Robol on retinal pigment epithelial cells and experimental proliferative vitreoretinopathy. Invest Ophthalmol Vis Sci 51: 3193-3204, 2010.

14. Je EM, Gwak M, Oh H, et al: Frameshift mutations of axon guidance genes ROBO1 and $\mathrm{ROBO} 2$ in gastric and colorectal cancers with microsatellite instability. Pathology 45: 645-650, 2013.

15. Sun H, Dai K, Tang T and Zhang X: Regulation of osteoblast differentiation by slit 2 in osteoblastic cells. Cells Tissues Organs 190: 69-80, 2009.

16. Smith-Berdan sec, Schepers K, Ly A, Passegue E and Forsberg EC: Dynamic expression of the Robo ligand Slit 2 in bone marrow cell populations. Cell Cycle 11: 675-682, 2012.

17. Kim KS, Kim JS, Lee MR, Jeong HS and Kim J: A study of microRNAs in silico and in vivo: Emerging regulators of embryonic stem cells. FEBS J 276: 2140-2149, 2009.

18. Fish JE, Wythe JD, Xiao T, et al: A Slit/miR-218/Robo regulatory loop is required during heart tube formation in zebrafish. Development 138: 1409-1419, 2011.

19. Choi E and Hwang KC: MicroRNAs as novel regulators of stem cell fate. World J Stem Cells 5: 172-187, 2013.

20. Guerit D, Brondello JM, Chuchana P, et al: FoxO3A regulation by miRNA-29a controls chondrogenic differentiation of mesenchymal stem cells and cartilage formation. Stem Cells Dev 23: 1195-1205, 2014

21. Lin X, Wu L, Zhang Z, et al: MiR-335-5p promotes chondrogenesis in mouse mesenchymal stem cells and is regulated through two positive feedback loops. J Bone Miner Res 29: 1575-1585, 2013

22. Yan C, Wang Y, Shen XY, et al: MicroRNA regulation ass ${ }^{\circ}$ Ciated chondrogenesis of mouse MSCs grown on polyhydroxyalkanoates. Biomaterials 32: 6435-6444, 2011.

23. Chen S, Yang L, Jie Q, et al: MicroRNA125b suppresses the proliferation and osteogenic differentiation of human bone marrowderived mesenchymal stem cells. Mol Med Rep 9: 1820-1826, 2014

24. Mao J, Lv Z and Zhuang Y: MicroRNA-23a is involved in tumor necrosis factor-alpha induced apoptosis in mesenchymal stem cells and myocardial infarction. Exp Mol Pathol, 2013. 